\title{
El impacto económico de la better regulation. Un análisis para España $^{1}$
}

\section{The economic impact of better regulation. An analisys for Spain}

\author{
Marisa Álvarez Suárez \\ Ministerio de Economía y Empresa (España) \\ marialuisa.alvarez@mineco.es
}

\section{NOTA BIOGRÁFICA}

Técnico Comercial y Economista del Estado. Licenciada en Economía por la Universidad de Oviedo. Máster en Relaciones Internacionales y Comunicación por la UCM. Actualmente Subdirectora de Unidad de Mercado, Mejora de la Regulación y Competencia. Líneas de especialización relacionadas con la promoción de la competencia, la regulación eficiente y el mercado interior de servicios.

\section{RESUMEN}

El impacto de las políticas de better regulation en relación con la productividad y crecimiento de una economía está ampliamente contrastado. En España, a pesar de los esfuerzos realizados en las últimas décadas, diferentes estudios e indicadores siguen señalando la persistencia de un potencial económico desaprovechado. En consecuencia, aún existe un margen de profundización y mejora en la reducción de cargas administrativas y regulatorias innecesarias y desproporcionadas y en el desarrollo de marcos regulatorios de calidad para el acceso y el ejercicio de las actividades económicas. Para ello, teniendo en cuenta el reparto de competencias entre las diferentes Administraciones públicas, las opciones de política económica deben focalizarse en el incremento de la cooperación interadministrativa, el intercambio de buenas prácticas y el fomento de los mecanismos de participación e iniciativa del sector privado.

\section{PALABRAS CLAVE}

Regulación eficiente; cargas administrativas; cooperación; productividad.

\begin{abstract}
The impact of better regulation policies in terms of productivity and economic growth is widely contrasted. In Spain, despite the regulatory efforts made in recent decades, studies and indicators reveal an important untapped potential for economic growth. Consequently, there is still room for improvement in the red tape reduction iniciatives and in the development of quality regulatory frameworks to facilitate access and exercise of economic activities. For this purpose, taking into account the distribution of competences among different Administrations, policy options should focus on the encouragement of inter-administrative cooperation, the exchange of best practices and the iniciatives to promote the involvement of civil society.
\end{abstract}

\section{KEYWORDS}

Smart regulation; red tape; cooperation; productivity.

1 Las opiniones expresadas en este artículo son responsabilidad exclusiva de su autora y no representan necesariamente las de ninguna institución. 


\section{SUMARIO}

1. INTRODUCCIÓN. 2. METODOLOGÍA. 3. LA RAZONABILIDAD ECONÓMICA DE LA BETTER REGULATION. 4. EL ANÁLISIS PARA ESPAÑA. 4.1. LA COMPLEJIDAD DEL MARCO REGULATORIO. 4.2. LOS EFECTOS EN LA PRODUCTIVIDAD Y EN EL CRECIMIENTO. 5. DISCUSIÓN: LOS AVANCES Y EL MARGEN DE MANIOBRA. 5.1. EL FOMENTO DE LA COOPERACIÓN INTERADMINISTRATIVA E INTERCAMBIO DE BUENAS PRÁCTICAS. 5.2. EL PAPEL DE LOS AFECTADOS. 6. CONCLUSIÓN. 7. REFERENCIAS BIBLIOGRÁFICAS.

\section{INTRODUCCIÓN}

Desde un punto de vista económico y jurídico la regulación es necesaria. La regulación ayuda a la reducción de costes de transacción, reduce los perjuicios y daños generados por los mercados que funcionan de forma ineficiente y permite la protección y el equilibrio de los posibles diferentes intereses públicos afectados. La regulación «cero» no suele ser la solución óptima y la mayoría de los mercados se caracterizan por la existencia de una regulación básica (laboral, fiscal, mercantil, etc.) y por, en su caso, una regulación específica o sectorial.

Sin embargo, a partir de un determinado umbral, la regulación -por innecesaria y desproporcionadase convierte en excesiva y redundante (conocida entonces como red tape) y genera costes relevantes en términos económicos y sociales.

Esta regulación ineficiente es un obstáculo para que los operadores puedan emprender o expandir su actividad económica y no tiene beneficios asociados. Esto supone un desincentivo a las inversiones empresariales y un coste macroeconómico relevante. Así, la mala regulación se puede llegar a convertir en un problema estructural de una economía y generar pérdida de competitividad, productividad, empleo y consecuentemente potencial de crecimiento.

En este artículo se realiza inicialmente una exposición de la razonabilidad teórica desde un punto de vista económico de la implementación de políticas orientadas a mejorar la regulación (a reducir o a eliminar dicha red tape) conocidas como políticas de better regulation tanto a nivel general como para España. Posteriormente se hace un análisis del potencial y del margen de maniobra para el fomento de la regulación eficiente en España y finalmente se analizan algunas de las posibles vías de actuación al respecto.

\section{METODOLOGÍA}

El objetivo de este trabajo es, inicialmente, a partir del análisis de la literatura y estudios académicos realizados en relación con variables microeconómicas específicas, resumir y recoger el impacto agregado económico de la better regulation. Así, partiendo de los trabajos realizados a nivel internacional que vinculan better regulation a variables económicas concretas, este artículo se enfoca, en su primera parte, en proporcionar una panorámica general y sistematizada de dicho impacto.

Una vez reflejado dicho impacto se pretende su particularización para el caso concreto de España. En este sentido, mediante el análisis de diferentes datos, fuentes e indicadores utilizados como "proxy» de la existencia de posibles problemas de complejidad regulatoria se sistematiza -a partir de la descripción de los diferentes estudios realizados al respecto- su posible incidencia sobre variables macroeconómicas como productividad o producto interior bruto. El objetivo de este análisis es intentar evaluar el posible margen económico existente y plantear las posibilidades de profundización y mejora en la parte final de discusión en donde se analizan algunas de las posibles vías, aún de relativo escaso desarrollo en España en comparación con otros países más avanzados en la materia, que podrían permitir una mejora en la solución del problema identificado.

La metodología empleada en la elaboración de este trabajo se ha basado fundamentalmente en el análisis de fuentes primarias y secundarias y en el tratamiento de determinados datos, encuestas de opinión e indicadores agregados. Estos datos e indicadores han sido utilizados con el objetivo de contrastar el impacto económico general y la obtención de los resultados específicos para España. En particular:

- Se ha procedido a la revisión, recopilación y estudio de diversos artículos y referencias documentales publicados por analistas y expertos en el tema tanto a nivel nacional como internacional.

- Se ha consultado diversa normativa de rango legal y reglamentario y otras actuaciones -programas, planes de actuación, convenios, memorias de análisis de impacto normativo etc.- a nivel de la Ad- 
ministración General del Estado.

- Se han analizado distintos informes y referencias documentales de diferentes instituciones públicas a nivel nacional e internacional y organismos privados de carácter estatal.

- Se ha procedido al tratamiento de diversos indicadores de mejora regulatoria y de productividad para España proporcionados por diversas instituciones internacionales con el objetivo de proceder a la comparación internacional en relación con otras economías desarrolladas.

- Se han analizado las prácticas regulatorias de diversos países considerados pioneros en la materia (Reino Unido, Alemania, Estonia, etc.) así como de la Comisión Europea a efectos de determinar las posibles vías de mejora.

\section{LA RAZONABILIDAD ECONÓMICA DE LA BETTER REGULATION}

La better regulation es un término de compleja definición que se ha ido concretando en el ámbito de la Organización de Cooperación y Desarrollo Económico (OCDE) y de la Unión Europea (UE) desde los años 90. La OCDE considera, en general, que la better regulation es una política que no tiene como finalidad reducir el número de normas sino aprobar las estrictamente necesarias, teniendo en cuenta que éstas deben ser proporcionadas a sus objetivos y ser claras, eficientes, accesibles y transparentes (OCDE, 2005). En este mismo sentido, para la UE, la better regulation es la política que busca mejorar y simplificar el entorno regulatorio y la regulación debe ser necesaria, apropiada, proporcionada, transparente, accesible y simple (Maldelkern, 2001). Ambas instituciones coinciden en señalar que la inversión de recursos en la mejora de la regulación produce beneficios tanto en el funcionamiento de la economía como en los ciudadanos.

En general, la necesidad de tomar medidas de impulso de creación de un marco eficiente de acceso y ejercicio para las actividades económicas como reflejo de esta better regulation y como iniciativas fundamentales para fomentar el crecimiento de una economía, está ampliamente respaldada por la teoría económica, las instituciones internacionales, los propios operadores e instituciones de análisis económico como, por ejemplo, las autoridades de defensa y promoción de la competencia.

Desde un enfoque económico, el beneficio para los ciudadanos y empresas de la better regulation o de la mejora de la regulación es clara. Las barreras y obstáculos innecesarios y desproporcionados generados por las malas prácticas regulatorias suponen una pérdida de oportunidades de crecimiento. Esto conlleva el desaprovechamiento de economías de alcance y de escala y la pérdida de recursos productivos en el estudio y el cumplimiento de dichas cargas. En consecuencia, se generan efectos negativos directos sobre la economía y el bienestar general.

Son múltiples los estudios realizados en las últimas décadas que señalan las bondades de la regulación eficiente en términos de productividad y crecimiento de una economía. En concreto, cabe señalar los siguientes:

- Las vinculaciones entre el nivel de regulación y el comportamiento económico agregado de un país partiendo del uso de indicadores de regulación han sido demostradas en diferentes estudios tanto con carácter sectorial como horizontal (Véase, por ejemplo: Nicoletti y Scarpetta, 2003; Bassanini y Ernst, 2002 o Schiantarelli, 2005). Estos estudios utilizan indicadores que diversas instituciones internacionales han construido y están utilizando como proxy del nivel de regulación de los países. Dichos indicadores se calculan partiendo de una metodología homogénea orientada a facilitar la comparabilidad internacional y temporal. Entre los diferentes indicadores cabría destacar, por su carácter horizontal, popularidad y cobertura internacional, los estimados por la OCDE conocidos como Indicadores PMR (Product Market Regulation), los Indicators of Regulatory Policy and Gobernance también de la OCDE y los calculados por el Banco Mundial (Doing Business Indicators).

- El impacto de la regulación sobre la productividad de las empresas y de una economía es un tema que ha atraído el interés de los expertos en las últimas décadas. La profusión de normas y la inconsistencia en su diseño causa confusión, retrasos e inseguridad jurídica imponiendo a las empresas costes excesivos en términos de recursos infrautilizados. Esto tiene un efecto directo sobre la productividad de las empresas y afecta en su conjunto, directa e indirectamente, a la productividad de una economía al impedir una correcta reasignación de los recursos productivos. El efecto del incremento de productividad asociado a un mercado mayor como resultado de la eliminación de las restricciones regulatorias es especialmente relevante en un contexto de mercados globalizados. Es decir, en un contexto internacional de apertura de mercados y globalización, los países compiten para atraer actividad económica también a través de la calidad y consistencia de su marco regula- 
torio. Como señalan numerosos estudios comparativos de las diferencias de productividad entre la UE y Estados Unidos (O'Mahoney y Van Ark, 2003; Skoczylas y Tissot, 2005; Denis et al., 2006 y Timmer et al., 2007, etc.) en economías abiertas, donde se incrementan las posibilidades de intercambio y las ganancias del mismo, la productividad es el principal determinante de la competitividad de un país a medio y largo plazo y, por tanto, del crecimiento real y potencial.

- El impacto del marco regulatorio ineficiente y de la fragmentación del mercado sobre la competencia en el mercado y consecuentemente sobre el crecimiento de una economía es también un efecto claramente contrastado y señalado por distintos expertos y autoridades de competencia. Por ejemplo, el Informe de la Agencia Sueca de Análisis de Políticas de Crecimiento (The Economic Effects of The Regulatory Burden - a Theoretical and Empirical Analysis), constata que un marco regulatorio complejo puede afectar negativamente a la competencia, desincentivando las inversiones y la incorporación de nuevos entrantes. Este efecto puede ser relativamente más acusado en los operadores y mercados con mayor capacidad innovadora, lo que provoca una asignación ineficiente de recursos que afectará a la capacidad de crecimiento de las empresas y de la economía en su conjunto.

En definitiva, los estudios realizados al respecto con carácter general para diferentes economías coinciden en señalar que, desde un punto de vista teórico y empírico, las barreras regulatorias innecesarias y desproporcionadas, los marcos normativos complejos, la existencia de duplicidades y la fragmentación del mercado consecuente, tiene un efecto microeconómico evidente sobre las empresas. Especialmente existe un efecto directo relevante en las pequeñas y medianas empresas y en su capacidad de crecimiento, teniendo en cuenta que el estudio del marco regulatorio lleva aparejada la necesidad de emplear recursos que podrían ser utilizados en actividades más productivas. Esta carencia genera incertidumbre e inseguridad jurídica e incluso obliga de facto a agentes sociales y a empresas a contratar ayuda externa con el objetivo de hacer frente a los procedimientos necesarios para poder realizar su actividad. Asimismo, afecta a las posibles sinergias derivadas de la diversificación de su actividad, incrementa los costes de sus consumos intermedios y su apertura a nuevas posibilidades de negocio (importante en relación con su capacidad de innovación y los nuevos modelos de negocio asociados a la revolución digital). En consecuencia, afecta a su capacidad de crecimiento y a su competitividad (Correa López, 2017).

A nivel macroeconómico todos estos efectos se traducen en un menor crecimiento de la economía en términos de Producto Interior Bruto (PIB), empleo y bienestar.

\section{EL ANÁLISIS PARA ESPAÑA}

\subsection{La complejidad del marco regulatorio}

La complejidad regulatoria viene vinculada, en general, a una cuestión de cantidad (las normas son demasiadas o se solapan) o de calidad (las normas son poco claras o están ambiguamente redactadas). En este sentido, la red tape en España puede estar relacionada no sólo a las posibles malas prácticas regulatorias y a la posible inercia de cierta «cultura sobreregulatoria» por parte de todas las Administraciones, sino también a la complejidad de nuestro sistema competencial. Así, en contextos caracterizados por la participación de diferentes autoridades competentes, la falta de una coordinación eficaz entre Administraciones puede generar duplicidades y solapamientos y marcos regulatorios inconsistentes o incoherentes.

La abundante producción normativa que se ha desarrollado en España, en diferentes ámbitos de todas las Administraciones Públicas (nacional, autonómico y local), ha creado un marco regulatorio que se suele considerar excesivamente oneroso y arduo para los operadores económicos por su dispersión y complejidad.

La complejidad normativa de España puede ser analizable mediante el estudio del crecimiento del número de normas aprobadas por las diferentes Administraciones públicas. Así, el siguiente cuadro gráfico refleja el número de normas aprobadas por las Comunidades Autónomas (CC.AA) y la Administración General del Estado entre 1978 y 2011. Este gráfico puede dar una idea y servir como aproximación de la tendencia al crecimiento de la intensidad regulatoria en España y, por tanto, del incremento de la complejidad regulatoria desde un punto de vista temporal ${ }^{2}$.

2 Los datos fueron obtenidos a partir de la publicación de normas en boletines oficiales de las CC.AA y del Boletín Oficial del Estado. En consecuencia, debe tenerse en cuenta que el cuadro mide la producción normativa estatal y autonómica pero no local y que no incluyen las normas no publicadas. Además, la estimación es conservadora y únicamente serviría para analizar la tendencia 
GRÁFICO I. NÚMERO DE NORMAS APROBADAS POR CC.AA Y AGE (1978-2011)

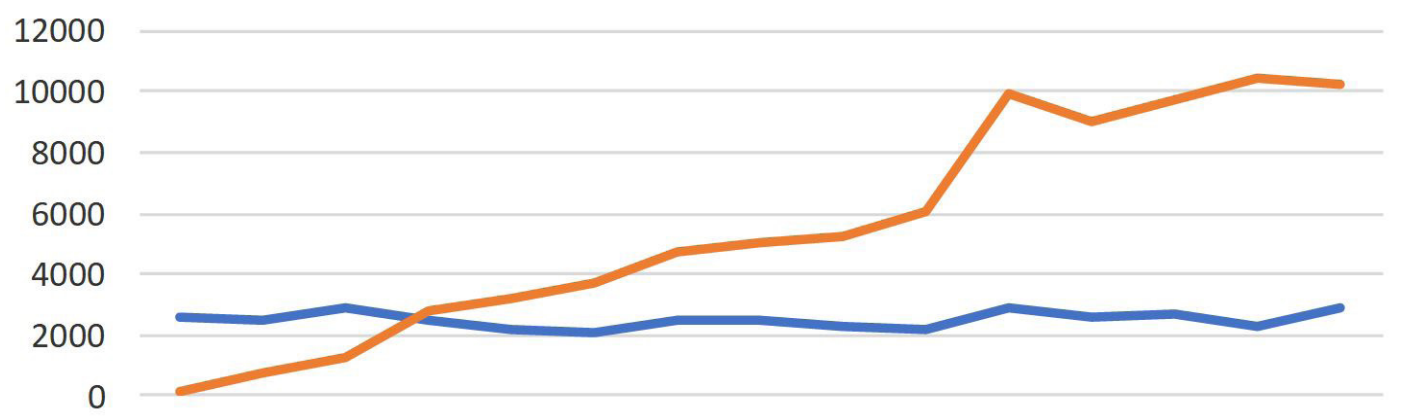

197819801982198419871990199319951997199920022004200620082011

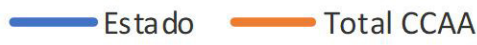

Fuente: Memoria de Análisis de Impacto Normativo del Anteproyecto de Ley de Garantía de la Unidad de Mercado.

Otros estudios realizados reflejan igualmente la existencia de dicha tendencia e intensidad regulatoria en España (en especial en comparación con otros países). Por ejemplo, en España se cumplimentan una media de más de 700.000 páginas anuales de contenido de los diversos boletines oficiales de las distintas Administraciones, 100 veces menos que Alemania (Marcos y Sántalo, 2010). En 2017, la Confederación Española de Organización Empresariales (CEOE) publicó el documento "La producción normativa en 2016" (documento actualizado en 2018 y 2019) que señala que las empresas españolas se enfrentan a un elevado grado de regulación que genera un complejo y denso marco normativo. Según este estudio se publicaron casi un millón (997.616) páginas en Boletines oficiales en 2018. A pesar del proceso de descentralización de competencias, la producción normativa del Estado se ha mantenido relativamente estable (aunque debe tenerse en cuenta la necesidad de realizar transposiciones de carácter básico de normativa comunitaria). Así, según estimaciones de este estudio de la CEOE, entre 1970 y 2018 a nivel del Estado se aprobaron 42.463 normas. Por su parte, la tendencia a la actividad regulatoria de las CC.AA se ha ido siempre incrementando. Debe además tenerse en cuenta que esta maraña normativa se ve ampliada por la regulación existente a nivel local y europeo (en especial, aquella de efecto directo). Especialmente, teniendo también en cuenta la evolución de la normativa local puede señalarse que con anterioridad de la aprobación de la Constitución Española se aprobaban menos de 3.000 normas al año mientras que en 2018 el número de normas se ha multiplicado por cuatro -11.737 normas en total- (Mora-Sanguinetti, 2019).

Las encuestas de opinión al respecto nos pueden proporcionar igualmente una buena aproximación de la percepción de los afectados en relación con la complejidad regulatoria de nuestro país. Por ejemplo, según una encuesta realizada por la Agencia Estatal de Evaluación de las Políticas Públicas y la Calidad de los Servicios (AEVAL) en 2011, entre un 60-67\% de los emprendedores consideran que para crear un negocio las Administraciones no están coordinadas y un $74 \%$ estiman que te preguntan por los mismos documentos varias veces (AEVAL, 2011). En este mismo sentido, el módulo de opinión sobre entorno empresarial incluido por el Instituto Nacional de Estadística en la Encuesta de Confianza Empresarial, hecho por primera vez público en marzo de 2014 , reflejaba que las empresas consideraban que la regulación económica $(51,6 \%)$ en España era el tercer factor del entorno empresarial -por detrás del entorno macroeconómico y la morosidad- con un impacto más desfavorable en su negocio en el 2013. Desde entonces la consideración de la regulación económica como factor desfavorable ha mejorado (siendo el primer factor de preocupación la demanda de los productos). Aun así, en 2018 la regulación económica continuaba siendo el tercer componente en nivel de importancia (33\%), por encima aún de factores como, por ejemplo, la fiscalidad, la disponibilidad de financiación o la eficiencia del mercado de trabajo. Debe entenderse que factores como la demanda de los productos, el entorno macroeconómico y la morosidad son cuestiones de tipo coyuntural mientras que la regulación económica lo es de tipo estructural. Además, en 2018, aproximadamente el $95 \%$ de los

en la medida en que únicamente se han contabilizado las normas en los años señalados. Es decir la contabilización se ha realizado únicamente para 15 años y no para los 32 de la serie. 
encuestados consideraban que los recursos destinados a hacer frente a los trámites de las diferentes Administraciones se mantenían estables o aumentaban y más del $45 \%$ consideraban que no percibían beneficios vinculados a la sustitución de autorizaciones por declaraciones responsables (cuestión vinculada a la mejora regulatoria) frente al $7 \%-8 \%$ que sí lo estaba percibiendo.

En definitiva, la complejidad del marco regulatorio de España puede considerarse relativamente elevada en términos cuantitativos. Las malas prácticas regulatorias y la complejidad normativa se ha visto históricamente reflejada en la desfavorable posición relativa de nuestra economía en los indicadores internacionales de regulación (los comentados PMR y Doing Business) donde España -a pesar de la mejora experimentada en los últimos años y teniendo en cuenta las limitaciones de estimación de estos indicadores (Mora-Sanguinetti y Matute, 2014; Mora-Sanguinetti y Salvador-Mora, 2016) - aún se sitúa en una posición relativa inferior al de otras economías desarrolladas (véase Tabla I). En consecuencia, por comparación, estos indicadores están reflejando, en definitiva, la existencia de problemas de tipo regulatorio en España y la posibilidad de mejora al respecto. En todo caso, cabría señalar que una potencial mejora del indicador PMR debe interpretarse únicamente como una mejora a nivel de la Administración central ${ }^{3}$.

TABLA I. CLASIFICACIÓN RELATIVA INDICADORES

\begin{tabular}{lcc}
\hline & Doing Business (2017) & PMR (2013) \\
\hline España & 28 & 18 \\
\hline Francia & 31 & 22 \\
\hline Italia & 46 & 9 \\
\hline Australia & 27 & 14 \\
\hline Reino Unido & 7 & 2 \\
\hline Canada & 17 & 17 \\
\hline Finlandia & 13 & 11 \\
\hline Alemania & 20 & 7 \\
\hline Irlanda & 18 & 19 \\
\hline EE.UU & 6 & 27 \\
\hline
\end{tabular}

Fuente: Elaboración propia a partir de los Indicadores PMR y DB de la OCDE y el Banco Mundial.

\subsection{Los efectos en la productividad y en el crecimiento}

Tal y como se ha comentado en el anterior epígrafe, a partir del análisis de diferentes indicadores, encuestas de opinión y comparativas internacionales es posible concluir que la complejidad normativa en España es relativamente elevada. Partiendo de dicha aproximación es posible, por tanto, analizar si -tal y como se reflejaba desde un punto de vista más general en el apartado 3 de este trabajo- dicha complejidad normativa tiene reflejo directo a nivel macroeconómico en términos, especialmente, de productividad y crecimiento de nuestra economía. Asimismo, cabría tener en cuenta la posible existencia de «costes indirectos» de dicha complejidad regulatoria vinculada a cuestiones como una mayor litigiosidad judicial o un mayor tamaño del sector público (Mora-Sanguinetti, 2019).

En este sentido, si bien durante los últimos treinta años y hasta la llegada de la crisis, el continuo crecimiento de PIB y del PIB per cápita han permitido la convergencia de la economía española con la media de la UE-15, existe consenso entre los economistas en considerar como principal debilidad histórica de la

${ }^{3}$ Los indicadores PMR solo recogen prácticas regulatorias a nivel estatal por lo que no podrían ser usados como proxy de la complejidad de los marcos normativos por la existencia de diferentes Administraciones con competencias regulatorias y sí como indicadores de regulación de la Administración Central. El Doing Business, sin embargo, si tiene en cuenta la existencia de diferentes Administraciones. A fecha de última revisión de este artículo se habían publicado ya los resultados del PMR 2018 en los que España se situaba en segunda posición empatado con Dinamarca y sólo por detrás de Reino Unido. 
economía española el lento crecimiento de la productividad desde un punto de vista estructural (Malo de Molina, 2005; Pérez, 2006; y Segura, 2006). Este relativo lento crecimiento de la productividad española puede analizarse a partir de los datos proporcionados al respecto para un conjunto amplio de países por la OCDE. Según datos de la OCDE, durante el promedio 1995-2017 el crecimiento de la productividad para la economía española (estimada como valor bruto añadido por hora trabajada a precios constantes) fue del 1,8. Un dato, en general, inferior a los de otras economías desarrolladas e inferior al promedio calculado para el conjunto de la Unión Europea (UE) de 28 Estados miembros.

GRÁFICO II. PROMEDIO PRODUCTIVIDAD (1995-2017)

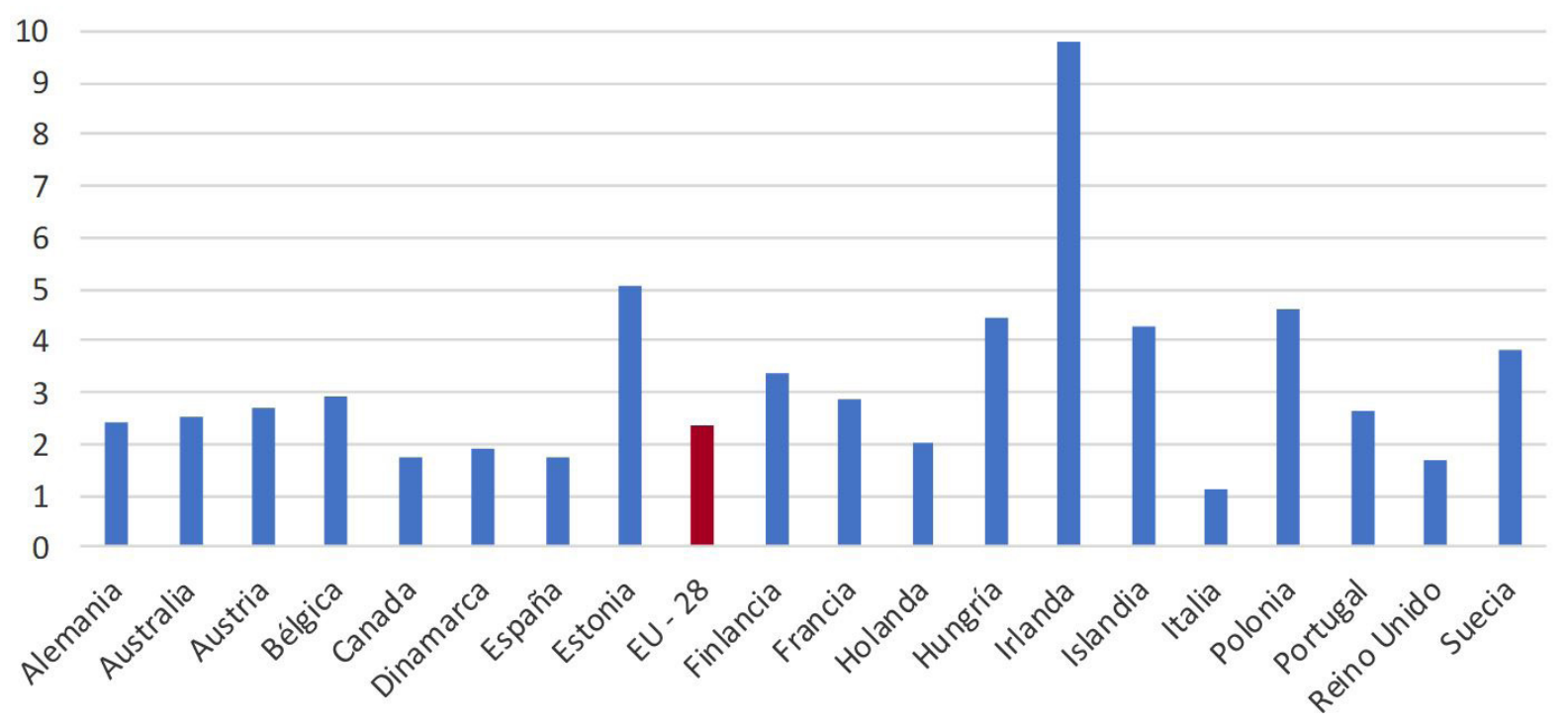

Fuente: Elaboración propia a partir de bases de datos OCDE Statistics.

Esta menor productividad relativa de la economía española puede deberse a una multitud de factores. No obstante, diversos análisis han concluido que, entre las diferentes posibles causas, cabría especialmente analizar la presencia de barreras regulatorias (Gual y Al., 2006). Esta vinculación entre complejidad regulatoria y productividad para la economía española se ha señalado con anterioridad en diversos estudios. Por ejemplo:

- Marcos y Santaló (2010) analizan el efecto medio de la intensidad regulatoria de las distintas regiones españolas sobre la productividad y la innovación. En particular, sus estimaciones apuntan a que la reducción de la productividad en la economía española entre los ochenta y mitad de la primera década de este siglo podría haberse evitado con una menor profusión regulatoria ${ }^{4}$. Además, encuentran que el impacto de la intensidad regulatoria incide de forma desigual a las empresas afectando relativamente más a aquellas más innovadoras, lo que genera una peor asignación de recursos.

- Asimismo, Zárate-Marco y Vallés-Giménez (2012) afirman que en España ha existido una tendencia expansiva de la producción normativa, producto del proceso de descentralización de competencias y a pesar de las reformas realizadas en las últimas décadas para la liberalización y el incremento de la competencia de los mercados. Su análisis refleja que la actividad regulatoria en las regiones españolas ha generado costes en términos de bajo crecimiento de la productividad.

En definitiva, el exceso de regulación, entendido éste como aquellas cargas administrativas y regulatorias al acceso y al ejercicio de la actividad económica de carácter innecesario y desproporcionado, parece haber tenido efectos directos sobre el crecimiento de la productividad de nuestra economía. Coherentemente con esto, los diferentes análisis realizados señalan que el coste del cumplimiento de dichas cargas tiene

4 Sus estimaciones muestran que un incremento del $100 \%$ en el número de normas se asocia a un decrecimiento de entre el 79 $81 \%$ del número de patentes regionales y con una más baja tasa de crecimiento de la productividad total de los factores de alrededor del $3,5 \%$. 
una importancia económica en términos de PIB muy destacable. Es decir, varios estudios han vinculado para España la existencia de complejidad regulatoria con menor crecimiento económico. Así:

- Kox (2005) en un análisis comparativo de la mayoría de Estados miembros de la UE estimó que para el caso de España el coste asociado a las cargas administrativas asciende al 4,6\% del PIB.

- López (2006) estimó que una reducción de cargas administrativas del 30\% para la economía española daría lugar a un aumento del PIB del 2,2\% en 10 años sobre el nivel que tendría en ausencia de shock.

- Gelauff y Lejour (2006), en un trabajo para el conjunto de países de la UE, estimó que el impacto en el largo plazo de una reducción de cargas administrativas del $25 \%$ en el caso de España sería equivalente a un incremento del PIB del 1,7\%, respecto al nivel que tendría sin esa reducción de cargas.

- En el "Inventario de las distorsiones de la unidad de mercado existentes en España" la CEOE en 2012 calculó el coste de la fragmentación del mercado por los diversos y complejos marcos regulatorios en 45.000 millones de euros, extrapolando para España los resultados que en su día hizo la Comisión Europea sobre la formación del Mercado Único en el denominado Informe Cecchini. En una posterior actualización de dicho estudio, se recogieron un total de 122 cargas administrativas detectadas por organizaciones de empresas territoriales y sectoriales.

En esta misma línea y con carácter general, para tener una aproximación del impacto económico que tiene la complejidad regulatoria en España, puede tomarse como indicador las estimaciones específicas calculadas en las políticas de mejora regulatoria ya implementadas a nivel estatal en la última década. Estas estimaciones suponen aproximaciones al impacto económico a largo plazo en términos de empleo y PIB -en un horizonte temporal de 10 años- que puede generar políticas orientadas a reducir la complejidad regulatoria. A la inversa, nos proporcionarían una estimación del «coste» de no implementar dichas políticas $y$, por tanto, de mantener el marco regulatorio a un nivel de complejidad dado. Las estimaciones han sido realizadas por los servicios económicos del Ministerio de Economía e incluidas en las Memorias de Análisis de Impacto Normativo de las normas o en los Programas Nacionales de Reforma (se incluye entre paréntesis el año de estimación). Para dichas estimaciones se ha usado el modelo de equilibrio dinámico Rational Expectations Model for Simulation (REMS), un modelo desarrollado en 2010 por el, entonces, Ministerio de Economía y Hacienda para la economía española que describe una economía pequeña y abierta con rigideces nominales y reales. Las estimaciones recogen el efecto acumulado de las reformas, es decir, la diferencia en el nivel de PIB o de empleo, a los 10 años de la implementación de cada medida en comparación con un escenario donde éstas no se hubieran llevado a cabo. Los análisis estiman un impacto agregado de entre el $0.31 \%$ del PIB (para reformas puntuales o sectoriales como las de la Ley 14/2013, de 27 de septiembre, de apoyo a los emprendedores y su internacionalización) al $1.63 \%$ del PIB para aquellas más ambiciosas y horizontales como las previstas en la Ley 20/2013, de 9 de diciembre, de garantía de la unidad de mercado.

TABLA II. IMPACTO ECONÓMICO DE REFORMAS DE BETTER REGULATION EN ESPAÑA

\begin{tabular}{llc}
\hline Reforma & PIB & Trabajo \\
\hline Reforma Administraciones públicas (2018) & 1,49 & 0,15 \\
\hline Ley Garantía Unidad de Mercado (2018) & 1,63 & 0,89 \\
\hline Transposición Directiva Servicios (2009) & 1,2 & $150.000-200.000$ \\
\hline Medidas de reducción de cargas de la Ley de Economía Sostenible - LES (2010) & 0,6 & - \\
\hline Medidas para las empresas de la LES (2010) & 0,77 & - \\
\hline Medidas no fiscales de la Ley de emprendedores (2016) & 0,31 & 0,15 \\
\hline
\end{tabular}

Fuente: Elaboración propia a partir de las Memorias de los Proyectos y de los Programas Nacionales de Reformas.

\footnotetext{
5 Este inventario identifica cuestiones, por ejemplo, en el ámbito del sector de alimentación y bebidas, del automóvil, en el sector de la construcción, en el sector farmacéutico, en el sector eléctrico, en el de tecnología sanitaria, en el de consultoría, en el comercio minorista, en el sector financiero y seguros, en instalaciones industriales, juego, publicidad, servicios para la dependencia, turismo, profesiones reguladas, profesionales técnicos, etc.
} 


\section{DISCUSIÓN: LOS AVANCES Y EL MARGEN DE MANIOBRA}

En el Consejo Europeo de primavera de 2007, el Gobierno español se comprometió a reducir las cargas administrativas derivadas de la legislación nacional en un $30 \%$ para el año 2012. Este objetivo se consideró sobradamente cumplido a partir de las estimaciones calculadas con el método simplificado para la reducción de cargas administrativas ${ }^{6} \mathrm{y}$, específicamente, con las medidas tomadas al respecto a partir de la transposición de la Directiva 2006/123/CE del Parlamento Europeo y del Consejo, de 12 de diciembre de 2006, relativa a los servicios en el mercado interior y con la aprobación de la Ley 2/2011, de 4 de marzo, de Economía Sostenible ${ }^{7}$.

Desde entonces otras muchas iniciativas - principalmente desde el punto de vista normativo- por parte de las diferentes Administraciones públicas han sido aprobadas con el objetivo de mejorar la regulación de las actividades económicas. Entre ellas cabría destacar, a nivel estatal, la aprobación de la Ley 20/2013, de 9 de diciembre, de garantía de la unidad de mercado, las medidas de reforma de la Administración pública o las iniciativas de fomento de la administración electrónica y de reducción de cargas de la Ley 39/2015, de 1 de octubre, del Procedimiento Administrativo Común de las Administraciones Públicas.

Los avances, por tanto, pueden considerarse importantes, al menos desde el punto de vista normativo, en la última década por parte de las diferentes Administraciones públicas. Este avance ha sido reflejado en los análisis realizados para España al respecto (OCDE, 2014).

Sin embargo, como se ha señalado, estos potenciales avances no parecen haber sido apreciados por los principales afectados -los operadores económicos y los agentes sociales- que siguen considerando que la regulación en España es demasiado compleja y excesiva (ver encuestas de opinión referidas en el apartado 4.1 de este artículo). En definitiva, teniendo en cuenta que, desde un punto de vista normativo, las posibles reformas están en su mayor parte realizadas, cabría preguntarse qué opciones de política económica quedan entonces para el fomento de la mejora de la regulación. En este sentido, atendiendo a las recomendaciones realizadas al respecto por instituciones internacionales ${ }^{8}$, y a la comparativa con diferentes países puede hacerse referencia a la necesidad de refuerzo de dos vías fundamentales: el fomento de la cooperación y de intercambio de buenas prácticas entre Administraciones y el papel activo de los operadores económicos para solventar los posibles problemas de implementación? .

\subsection{El fomento de la cooperación interadministrativa e intercambio de buenas prácticas}

En un país con un reparto competencial entre diferentes Administraciones públicas el margen de mejora pasa, indisolublemente, por el fomento de la cooperación y el intercambio de buenas prácticas entre Administraciones. Cuando las competencias se encuentran distribuidas entre distintos niveles administrativos, la falta de cooperación entre Administraciones públicas se puede traducir, como se ha señalado, en marcos regulatorios complejos e incoherentes que pueden suponer una deficiencia estructural del sistema económico. En definitiva, no es sólo necesario que cada Administración avance de forma individual en la better regulation sino que lo hagan de forma coordinada para generar marcos normativos óptimos. Esta necesidad de cooperación entre Administraciones en aras del impulso de la better regulation ha sido tam-

\footnotetext{
6 Véase para una mayor explicación de este método la "Guía Metodológica para la elaboración de la Memoria del Análisis de Impacto Normativo" disponible en: http://www.minhafp.gob.es/AreasTematicas/Gobernanza/simplifica/guia_metodologica_ain.pdf.

7 Como continuación de este proceso la Comisión Europea adoptó, el 2 de octubre de 2013, una Comunicación sobre «Adecuación y eficacia de la normativa: resultados y próximas etapas», conocida como "REFIT, Regulatory Fitness and Perfomance" en la que se establece como uno de sus objetivos la reducción continua de las cargas administrativas que afectan a las empresas -especialmente a las pequeñas y medianas empresas-.

8 Véase al respecto las últimas recomendaciones realizadas a España en el marco del Semestre Europeo vinculadas a la implementación de la LGUM (disponible las del año 2018 en https://ec.europa.eu/info/publications/2018-european-semester-country-specificrecommendations-council-recommendations_es) o los Informes del artículo IV del FMI. (disponible el del año 2018 en https://www. imf.org/en/Publications/CR/Issues/2018/11/21/Spain-2018-Article-IV-Consultation-Press-Release-Staff-Report-and-Statement-by-theExecutive-46381).

9 Para el impulso de la mejora de la regulación no debe tampoco obviarse la importancia de otros instrumentos de carácter más general orientados a la «modificación» de la cultura regulatoria y administrativa como pueden ser por ejemplo los cursos de formación y difusión específicos para los empleados públicos encargados del diseño y ejecución de las normas y el fomento y desarrollo de los diferentes instrumentos de mejora de la regulación (evaluación ex post y ex ante, reglas de reducción de cargas tipo one in one out) (Álvarez, 2017).
} 
bién señalada a nivel internacional con carácter general (OCDE, 2018) y muy especialmente para España (OCDE, 2014).

Si bien, como se ha comentado, las Administraciones han avanzado desde un punto de vista normativo y en el marco cada una de sus competencias en aras de la mejora de la regulación, el margen para el intercambio de buenas prácticas y la cooperación aún es grande en España. Así:

- El estudio publicado en 2011 por la extinta AEVAL, puso de manifiesto cómo entre las distintas CC.AA españolas existen disparidades importantes en las materias regulatorias que afectan al desarrollo de la actividad económica y, por tanto, en las cargas administrativas que éstas imponen. Bajo el supuesto de convergencia a la media nacional de los costes de creación de empresas en las regiones cuyos costes están por encima de la media, y manteniendo en su nivel actual los costes de las regiones que están por debajo de la media, se ha estimado la posibilidad de una reducción del $35 \%$ de las cargas administrativas asociadas a la creación de empresas.

- En el año 2015, el Banco Mundial publicó el informe "Doing Business in Spain 2015" que desagrega y compara a nivel subnacional, la regulación a la que tienen que hacer frente las empresas españolas en las diferentes Comunidades Autónomas y Entes Locales. Usando la metodología básica del Indicador del Doing Business, el Banco Mundial estimó diferentes indicadores de carácter subnacional que pusieron de manifiesto la existencia de solapamientos entre diferentes administraciones territoriales; la necesidad de una mayor coordinación entre ellas; la revisión de la figura de la autorización por otras menos restrictivas como la declaración responsable; y la necesidad de ahondar en la simplificación de cargas para los operadores. Los resultados de dicho estudio concluyeron que La Rioja, la Comunidad de Madrid y Navarra serían las CC.AA donde existe mayor facilidad para emprender un negocio. No obstante, el estudio identificó que en todas las CC.AA existen buenas prácticas en diversas subáreas del análisis y que existe, por tanto, una heterogeneidad importante en la regulación. Esto implica que existe un margen de maniobra relevante para la cooperación y la mejora.

El intercambio de buenas prácticas y el papel de la cooperación interadministrativa es vital para la eliminación de duplicidades y solapamientos, para clarificar y simplificar los marcos regulatorios aplicables a las actividades económicas y, en consecuencia, avanzar en la mejora regulatoria. En este ámbito, a nivel español, asumen una especial relevancia los órganos de cooperación (Conferencias sectoriales u otros órganos o grupos de trabajo de carácter interadministrativo) y el papel de Estado como Administración impulsora de su funcionamiento. Tanto la Ley 20/2013, de 9 de diciembre, de garantía de la unidad de mercado (artículo 12) como la Ley 40/2015, de 1 de octubre, de Régimen Jurídico del Sector Público (artículo 147 y siguientes) han supuesto un refuerzo jurídico de la regulación y funciones de estos organismos. Sin embargo, la actividad de estos órganos -en términos de acuerdos adoptados o incluso de reuniones convocadas- sigue siendo, en general, escasa y, en consecuencia, las posibilidades de mejora en su funcionamiento aún amplias. Así, por ejemplo, las más de 50 Conferencias sectoriales, u órganos de carácter equivalente, constituidas han mantenido una media total de 62 reuniones al año durante el periodo 20122016, es decir, ni siquiera una media de dos reuniones al año por órgano (Ministerio de Presidencia y para las Administraciones Territoriales, 2017).

Asimismo, la Administración electrónica abre nuevas vías de comunicación para las Administraciones (entre ellas y con el ciudadano) y, en consecuencia, incrementa las oportunidades de mejorar el funcionamiento interno del sector público y la regulación (Maudes y Silos, 2016). Debe, por tanto, impulsarse desde un punto de vista material y ejecutivo (y no sólo normativo, cuestión que se puede considerar totalmente realizada con la aprobación de la Ley 39/2015, de 1 de octubre) el uso los medios digitales más avanzados por parte de la Administración.

El intercambio de buenas prácticas entre Administraciones no tiene que circunscribirse al ámbito regional o local. En el ámbito de la Unión Europea y, especialmente, en el de la OCDE (en foros como por ejemplo el Comité de Política Regulatoria) se ha trabajado profusamente en el conocimiento y difusión por parte de los Estados miembros de las diferentes prácticas desde el punto de vista institucional y regulatorio que se consideran de relevancia en el ámbito de la mejora de la regulación. Cabría destacar en este sentido el reciente informe de la OCDE "Better Regulation Practices across de European Union" que proporciona abundante información sobre el posible margen de profundización de España en los diferentes ámbitos de la mejora de la regulación por comparación con otros países europeos. Teniendo en cuanta los diferentes indicadores señalados en dicho informe, España tendría margen de mejora claro en la evaluación ex ante (lejos 
aún de prácticas ${ }^{10}$ de países pioneros en la materia como Reino Unido, Alemania y Estonia). Muy especialmente también, a pesar de los recientes avances normativas al respecto, España aún podría avanzar en la implementación de la evaluación ex post de las normas (donde, según el comentado informe, se encuentra claramente por debajo de la media europea) mediante la implantación de prácticas que han sido ya llevadas a cabo en países como Reino Unido, Italia o Alemania ${ }^{11}$.

\subsection{El papel de los afectados}

La participación de los afectados en el proceso de identificación de cargas administrativas y de fomento de mejora de la regulación es igualmente una pieza clave para la remoción de obstáculos innecesarios y desproporcionados a la actividad económica. De esta forma se permite un enfoque "práctico» basado en los problemas reales y en la evidencia algo que es considerado especialmente relevante para avanzar en la mejora regulatoria (OCDE, 2019).

En esta línea, la Administración debe proporcionar instrumentos a los operadores económicos para que su voz sea oída y escuchada en el ámbito de la mejora regulatoria. Por ejemplo, un instrumento de utilidad puede ser la firma de convenios con las principales entidades representativas para la identificación y remoción de cargas. En este sentido, desde 2008 la CEOE-CEPYME y otras asociaciones han colaborado con el Ministerio de Administraciones públicas mediante la firma, en su caso, de sucesivos convenios para la identificación de cargas ${ }^{12}$. El trabajo debe focalizarse no sólo en la identificación y catalogación de las cargas administrativas y regulatorias, sino también en la búsqueda de propuestas de solución de las mismas y en la evaluación «expost» de los resultados de dichos acuerdos, cuestión donde, de nuevo, la cooperación entre administraciones supone un factor fundamental. En esta línea, puede igualmente tenerse en cuenta la existencia de buenas prácticas de países pioneros en la materia al respecto, como, por ejemplo, en Dinamarca la creación del "Denmark's Business Forum for Better Regulation" ${ }^{13}$. Este foro formado por representantes de operadores económicos y Administración lleva desde 2012 trabajando de forma sistematizada y coordinada para la reducción de cargas administrativas y mejora del clima de negocios.

Como mecanismos de especial relevancia orientados a dar voz a los operadores económicos y a reforzar su papel o rol clave como impulsores de la mejora regulatoria cabría señalar aquellas vías de carácter jurídico-administrativo "privilegiadas» que se han puesto en marcha recientemente en normas como, por ejemplo, la Ley 19/2013, de 9 de diciembre, de transparencia, acceso a la información pública y buen gobierno o la Ley 20/2013, de 9 de diciembre de garantía de la unidad de mercado. Estos nuevos procedimientos, basados en otros ya existentes a nivel europeo, se configuran como vías de disciplina del comportamiento de la Administración pública. Se evita con ellos que las intenciones de mejora regulatoria queden plasmadas únicamente de forma programática en las normas, poniendo a disposición de la sociedad civil ventanillas especiales para denunciar actuaciones de las Administraciones que se consideren contrarias a determinados principios normativos de la better regulation. De esta forma, se obliga a las Administraciones públicas al efectivo cumplimiento de dichos principios solventando un posible «déficit» de implementación de la normativa (es decir, cuando la norma no se aplica o se aplica ineficientemente). El funcionamiento de estos mecanismos debe ser, por tanto, reforzado e impulsado (Alvarez y Balsa, 2017).

Por último, en España, la implicación de los interesados en el proceso normativo es un camino a estudiar como vía para el impulso de la mejora de la regulación. Se han hecho grandes avances recientemente como, por ejemplo, con la instauración de la denominada consulta pública previa de los proyectos normati-

10 Por ejemplo, la realización de evaluaciones ex ante internos de normativa comunitaria en negociación, la respuesta obligatoria de las observaciones recibidas relativas al impacto en los procesos de consulta pública, etc.

11 Por ejemplo, mediante la elaboración de Guías y sistematización de procedimientos para la evaluación expost y la definición de criterios claros.

12 Como resultado de esta colaboración la CEOE ha publicado diversos informes. Por ejemplo, en 2013 la CEOE emitió el informe de las cargas administrativas soportadas por las microempresas, de menos de cinco trabajadores y los empresarios autónomos españoles, que suponen el 92,4\% del total de empresas del país. En este caso, se identifican dieciocho medidas correspondientes a siete ministerios y un Organismo Autónomo estatal que ahorrarían en conjunto 320 millones de EUR al año. Asimismo, la CEOE y Cepyme en 2014 publicaron el estudio de "Las cargas administrativas soportadas por las empresas derivadas de la fragmentación del mercado interior". El estudio identifica 36 cargas administrativas correspondientes a cinco ministerios, que de reducirse supondrían un ahorro para la Administración Española y las empresas de 1.770 millones de euros. En 2015 como continuación y complementación de este primer documento se realizó el estudio "Las cargas administrativas soportadas por las empresas derivadas de la fragmentación del mercado interior II" en el que se exponen 24 propuestas de simplificación correspondientes a cinco ministerios.

13 Más información en https://danishbusinessauthority.dk/business-forum-better-regulation. 
vos en la Ley 39/2015, de 1 de octubre. No obstante, siguen existiendo ámbitos de la vida pública donde la participación de los potenciales interesados es escasa o nula. Así, siguiendo prácticas de países pioneros en la materia basadas en el principio de gobierno abierto, como por ejemplo Reino Unido o Eslovenia o el propio funcionamiento de la Comisión Europea, es posible incrementar la intervención de los operadores en cuestiones de su interés. Por ejemplo, fomentando su participación en procesos de consulta pública realizados a nivel comunitario, en la negociación de normativa comunitaria o en los procesos de evaluación expost de las normas.

\section{CONCLUSIÓN}

Uno de los objetivos de toda política económica debe ser el crear un entorno favorable para que los operadores se beneficien de un marco regulatorio eficiente para el acceso y ejercicio de sus actividades económicas, asumiendo siempre que otros posibles intereses públicos afectados quedan convenientemente salvaguardados. Los estudios demuestran que la regulación ineficiente tiene un impacto claro desde el punto de vista micro y macroeconómico. Por ello, la conocida como better regulation, conjunto de principios e instrumentos de intervención pública orientados a la consecución de los objetivos previstos al mínimo coste, se convierte en una política pública de indispensable fomento para una economía.

En el caso de España, a pesar de las diferentes iniciativas implementadas en las últimas décadas básicamente desde un punto de vista normativo, los diferentes indicadores de mejora regulatoria y estudios realizados, así como, en general, la percepción de los operadores económicos y los agentes sociales parecen indicar que aún existe un potencial económico desaprovechado. Esto podría ser debido, entre otras causas, a la existencia de un posible «déficit» de implementación (la norma existe, pero no se aplica o se aplica de forma ineficiente) o por la existencia de marcos de regulación complejos, inconsistentes y dispersos, producto de la intervención descoordinada de diferentes autoridades competentes (es decir, no se trataría únicamente de que cada Administración avance de forma individual sino de que lo hagan de forma coordinada).

En definitiva, el margen de maniobra en países con competencias compartidas entre diferentes Administraciones públicas pasa, entre otras iniciativas, por el fomento y el refuerzo del funcionamiento de los diferentes mecanismos de cooperación interadministrativa existentes. Asimismo, para solventar los posibles «déficits» de implementación de la normativa y dar voz a la sociedad civil en estos procesos son igualmente importantes los mecanismos de comunicación, interacción y resolución de problemas con los agentes sociales y operadores afectados. En este sentido, el análisis de las buenas prácticas de otras Administraciones es un instrumento útil para identificar las vías de mejora y los instrumentos que pueden ser implementados en favor de la mejora regulatoria. Especialmente, a nivel internacional, países considerados pioneros en la materia como Reino Unido, Alemania o Eslovenia (así como la propia Comisión Europea) han implementado determinadas prácticas en cuestiones como la evaluación ex ante y ex post de las normas y la participación de los interesados que podrían ser analizadas y, en su caso, replicadas, en el ámbito español.

\section{REFERENCIAS BIBLIOGRÁFICAS}

AGENCIA ESTATAL DE EVALUACIÓN DE LAS POLÍTICAS PÚBLICAS Y LA CALIDAD DE LOS SERVICIOS (2011): Evaluación de las trabas administrativas para la creación de empresas: segundo informe sobre los trámites administrativos para la creación de empresas. Disponible en web: http://www.aeval.es/export/sites/aeval/comun/ pdf/evaluaciones/E31-Trabas.pdf.

AGENCIA SUECA DE ANÁLISIS DE POLÍTICAS DE CRECIMIENTO (2010): The Economic Effects of The Regulatory Burden - a Theoretical and Empirical Analysis. Disponible en web: http://www.tillvaxtanalys. se/downloa d/18.201965214d8715afd13d8c6/1432670893004/Report_2010_14.pdf.

ÁLVAREZ SUÁREZ, M. (2017): "La mejora de la regulación en España como política pública: análisis y evolución”, en Gestión y Análisis de Políticas Públicas. Nueva época, núm. 17, págs. 26-39. Disponible en web: https:// revistasonline.inap.es/index.php/GAPP/article/view/10413/11041. DOI: https://doi.org/10.24965/gapp.v0i17.10413.

ÁLVAREZ SUÁREZ, M., BALSA PASCUAL, C. (2017): "Mecanismos de la Ley de Garantía de la Unidad de Mercado. La buena regulación al alcance de los operadores", en GUILLÉN CARAMÉS, J., CUERDO MIR, M. (dirs.), RAMS RAMOS, L. (ed.): Estudios sobre la Ley de Garantía de la Unidad de Mercado. Madrid: Thomson Aranzadi.

BASSANINI, A., ERNST, E. (2002): "Labour market institutions, product market regulation, and innovation: crosscountry evidence", en OECD Economics Department Working Papers, núm. 316. Disponible en web: https://www. 
oecd-ilibrary.org/docserver/002243151077. pdf?expires=1528802970\&id=id\&accname=guest\&checksum=FB56FC 3688EA1618CE84DC723E3562F2. DOI: https://doi.org/10.1787/002243151077.

BOSCÁ, J. E., DOMÉNECH, R., FERRI, J., VARELA, J. (2011): The Spanish Economy: A general equilibrium perspective. Reino Unido: Palgrave Macmillan.

CEOE (2012): Inventario de las distorsiones de la unidad de mercado existentes en España. Disponible en web: http:// www.cen7dias.es/BOLETINES/422/CEOE_distorsiones_unidad_mercado.pdf.

CEOE (2019): La producción normativa en 2018. Disponible en web: https://www.ceoe.es/es/informes/Actividad_ Economica/la-produccion-normativa-en-2017.

CEOE-CEPYME (2012): Informe de las cargas administrativas soportadas por las microempresas y los empresarios autónomos españoles. Disponible en web: http://contenidos.ceoe.es/resources/image/cargas_administrativas_ microempresas_autonomos_2012.pdf.

CEOE-CEPYME (2014): Las cargas administrativas soportadas por las empresas derivadas de la fragmentación del mercado interior. Disponible en web: https://contenidos.ceoe.es/CEOE/var/pool/pdf/publications_docs-file-21cargas-administrativas-derivadas-de-la-fragmentacion-del-mercado-interior-espanol-ii.pdf.

CEOE-CEPYME (2015): Las cargas administrativas soportadas por las empresas derivadas de la fragmentación del mercado interior II. Disponible en web: https://www.ceoe.es/es/informes/Actividad_Economica/cargasadministrativas-derivadas-de-la-fragmentacion-del-mercado-interior-espanol-ii.

COMISIÓN EUROPEA (2001): Informe MaldeKern sobre Mejora de la Regulación. Bruselas. Disponible en: https:// www.parlament.cat/document/intrade/28773.

CORREA-LÓPEZ, M. (2017): Regulación de los servicios, precios de los consumos intermedios y exportaciones. Madrid: Banco de España (Artículos Analíticos). Disponible en web: https://www.bde.es/f/webbde/SES/Secciones/ Publicaciones/InformesBoletinesRevistas/ArticulosAnaliticos/2017/T1/fich/beaa1701-art5.pdf.

DENIS, D. J., HANOUNA, P., SARIN, A. (2006): "Is there a dark side to incentive compensation?", en Journal of Corporate Finance, vol. 12, núm. 3, págs. 467-488. Disponible en web: http://citeseerx.ist.psu.edu/viewdoc/downlo ad?doi=10.1.1.458.4441\&rep=rep1\&type=pdf. DOI: $h$ ttps://doi.org/10.1016/j.jcorpfin.2005.08.006.

GELAUFF, G. M., LEJOUR, A. M. (2006): "Five Lisbon Highlights: The Economic Impact of Reaching these Targets", en CPB Document, núm. 104. CPB Netherlands Bureau for Economic Policy Analysis. Disponible en web: https://www.cpb.nl/sites/default/files/publicaties/download/five-lisbon-highlights-economic-impact-reachingthese-targets.pdf.

GUAL, J., JÓDAR ROSELL, S., RUIZ POSINO, A. (2006): "El problema de la productividad en España: ¿Cuál es el papel de la regulación?", en Documentos de Economía «la Caixa», núm. 1. Disponible en web: http://www. caixabankresearch.com/documents/10180/51459/de01_esp.pdf.

INSTITUTO NACIONAL DE ESTADÍSTICA (INE) (2019): Indicadores de confianza empresarial. Módulo sobre entorno empresarial y buena regulación. Serie 2013-2018. Disponible en web: http://www.ine.es/dynt3/inebase/es/ index. $h$ tm? path=/t37/p199/p01/serie/\&dh=0\&capsel=0.

LÓPEZ, P., ESTRADA, A., THOMAS, C. (2008): “Una primera estimación del impacto económico de una reducción de las cargas administrativas en España”, en Boletín económico, núm. julio-agosto, págs. 81-92. Madrid: Banco de España. Disponible en web: https://www.bde.es/f/webbde/SES/Secciones/Publicaciones/ InformesBoletinesRevistas/BoletinEconomico/08/JulAgo/Fich/art1.pdf.

MALO DE MOLINA, J. L. (2005): "Una larga fase de expansión de la economía española", en Documentos Ocasionales, núm. 0505. Madrid: Banco de España. Disponible en web: https://www.bde.es/f/webbde/SES/ Secciones/Publicaciones/PublicacionesSeriadas/DocumentosOcasionales/05/Fic/do0505.pdf.

MARCOS, F., SANTALÓ, J., (2010): "Regulation, innovation and productivity", en IE Business School Working Paper, WP10-04. Disponible en web: http://www.cen7dias.es/BOLETINES/422/instituto_empresa.pdf.

MAUDES GUTIÉRREZ, A., SILOS RIBAS, M. (2016): "Una regulación económica más eficiente en el mundo digital. Las autoridades independientes de productividad”, en Información Comercial Española, ICE: Revista de Economía, núm. 891, págs. 61-80. Disponible en web: http://www.revistasice.com/index.php//CE/article/view/1865. DOI: $h$ ttps://doi.org/10.32796/ice.2016.891.1865.

MINISTERIO DE ECONOMÍA Y COMPETITIVIDAD (2013): Memoria de Análisis de Impacto Normativo del Anteproyecto de Ley de Garantía de la Unidad de Mercado.

MINISTERIO DE ECONOMÍA Y HACIENDA (2010): Informe sobre la transposición de la Directiva de Servicios. Madrid: Ministerio de Economía y Hacienda. Disponible en web: http://www.mineco.gob.es/stfls/mineco/economia/ enlaces/destacados/Doc3DS.pdf.

MINISTERIO DE HACIENDA Y FUNCIÓN PÚBLICA (2019): Programas Nacionales de Reformas. Madrid: Ministerio de Hacienda y Función Pública. Disponible en web: http://www.minhafp.gob.es/es-ES/CDI/Paginas/ EstrategiaPoliticaFiscal/ProgramaNacionalReformas.aspx.

MINISTERIO DE LA PRESIDENCIA Y PARA LAS ADMINISTRACIONES TERRITORIALES (2017): Informe sobre la Actividad de las Conferencias Sectoriales 2016. Disponible en web: http://www.seat.mpr.gob.es/dam/es/portal/ areas/politica_autonomica/coop_autonomica/Conf_Sectoriales/Documentacion/Conf_Sect_anuales/parrafo/0/text_ es_files/INFORME_CC_SS_2016.pdf. 
MORA-SANGUINETTI, J. S. (2019): "La «complejidad» de la regulación española ¿cómo medirla? ¿qué impacto económico tiene?”, en Información Comercial Española, ICE: Revista de Economía, núm. 907, págs. $147-162$. DOI: $h$ ttps://doi.org/10.32796/ice.2019.907.6784.

MORA-SANGUINETTI, J. S., MARTÍNEZ MATUTE, M. (2014): "La regulación en el mercado de productos en España según los indicadores de la OCDE”, en Boletín Económico, vol. 12/2014, págs. 45-54. Madrid: Banco de España. Disponible en web: https://www.bde.es/f/webbde/SES/Secciones/Publicaciones/InformesBoletinesRevistas/ BoletinEconomico/14/Dic/Fich/be1412.pdf.

MORA-SANGUINETTI, J. S., SALVADOR-MORA, S. (2016): "El impacto económico de la actividad normativa. Los riesgos de la "gran convergencia»", en Revista de Economia Industrial, núm. 398, págs. 117-128. Disponible en web: http://www.mincotur.gob.es/Publicaciones/Publicacionesperiodicas/Economialndustrial/ RevistaEconomialndustrial/398/MORA\%20SANGUINETTI\%20Y\%20SALVADOR\%20MORA.pdf.

NICOLETTI, G., SCARPETTA, S. (2003): "Regulation, productivity and growth: OECD evidence", en Economic policy, vol. 18, núm. 36, págs. 9-72. Disponible en web: https://academic.oup.com/economicpolicy/articleabstract/18/36/9/402823. DOI: https://doi.org/10.1111/1468-0327.00102.

O'MAHONEY, M., VAN ARK, B. (2003): Eu productivity and competitiveness: an industry perspective. Can Europe resume the catching-up process?. Luxembourg: European Commission. Disponible en web: https://www.rug.nl/ ggdc/docs/eu_productivity_and_competitiveness.pdf.

OECD (2008): OECD Guiding Principles for Regulatory Quality and Performance. Paris: OECD Publishing. Disponible en web: http://www.oecd.org/fr/reformereg/34976533.pdf. DOI: https://doi.org/10.1787/9789264056381-en.

OECD (2014): Spain: From Administrative Reform to Continuous Improvement. Paris: OECD Publishing. DOI: https:// doi.org/10.1787/9789264210592-en.

OECD (2018): OECD Regulatory Policy Outlook 2018. Paris: OECD Publishing. DOI: https://doi. org/10.1787/9789264303072-en.

OECD (2019): Better Regulation Practices across the European Union. Paris: OECD Publishing. DOI: https://doi. org/10.1787/9789264311732-en.

PÉREZ GARCÍA, F., MAUDOS VILLARROYA, J., PASTOR MONSÁLVEZ, J. M., SERRANO MARTíNEZ, L. (2006): Productividad e internacionalización. El crecimiento español ante los nuevos cambios estructurales. Bilbao: Fundación BBVA. Disponible en web: https://w3.grupobbva.com/TLFU/dat/DE_2006_IVIE_productividad_e_ internacionalizacion.pdf.

SCHIANTARELLI, F. (2005): "Product market regulation and macroeconomic performance: A review of cross-country evidence", en World Bank Publications, vol. 1.791. Disponible en web: http://fmwww.bc.edu/ec-p/wp623.pdf.

SEGURA, J. (coord.) (2006): La productividad en la economía española. Madrid: Fundación Ramón Areces.

SKOCZYLAS, L., TISSOT, B. (2005): "Revisiting Recent Productivity Developments across OECD Countries", en BIS Working papers, núm. 182. Basel (Switzerland): Bank for International Settlements (BIS). Disponible en web: https://www.bis.org/publ/work182.pdf.

SUBNATIONAL DOING BUSINESS (2015): Doing business en España 2015. Disponible en web: http://espanol. doingbusiness.org/reports/subnational-reports/spain.

TIMMER, M., O'MAHONY, M., VAN ARK, B. (2007): "Growth and Productivity Accounts from EU KLEMS: An Overview”, en National Institute Economic Review, vol. 200, núm. 1, págs. 64-78. Disponible en web: http://www. euklems.net/data/overview_07i.pdf. DOI: https://doi.org/10.1177/0027950107080390.

ZÁRATE-MARCO, A., VALLÉS-GIMÉNEZ, J. (2012): "The cost of regulation in a decentralized context: the case of the Spanish regions", en European Journal of Law and Economics, vol. 33, núm. 1, págs. 185-203. Disponible en web: https://link.springer.com/article/10.1007/s10657-010-9154-2. DOI: https://doi.org/10.1007/s10657-010-9154-2. 